\title{
Clinical Relevance of Pharmacist Intervention: Development of a Named Entity Recognition Model on Unstructured Comments
}

\author{
Justine CLARENNE,b,c,1, Sonia PRIOU ${ }^{\mathrm{d}}$, Aymeric ALIXE ${ }^{\mathrm{d}}$, Olivier MARTIN ${ }^{\mathrm{d}}$, \\ Céline MONGARET ${ }^{\mathrm{a}, \mathrm{b}}$ and Pierrick BEDOUCH ${ }^{\mathrm{c}, \mathrm{e}}$ \\ a Pharmacy Department, Reims Champagne-Ardenne University, Reims, France \\ ${ }^{\mathrm{b}}$ Pharmacy Department, Reims University Hospital, Reims, France

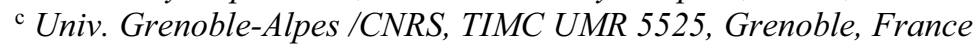 \\ d Lumio Medical, Paris, France \\ e Pharmacy Department, Grenoble University Hospital, La Tronche, France
}

\begin{abstract}
We developed a clinical named entity recognition model to predict clinical relevance of pharmacist interventions (PIs) by identifying and labelling expressions from unstructured comments of PIs. Three labels, drug, kidney and dosage, had a great inter-annotator agreement $(>60 \%)$ and could be used as reference labelization. These labels also showed a high precision $(>70 \%)$ and a variable recall (50-90\%).
\end{abstract}

Keywords. Natural Language Processing; clinical NER; clinical pharmacy; drugrelated problem

\section{Introduction}

During pharmaceutical care activities, pharmacist interventions (PIs) are formulated using a standardization instrument integrating pre-defined categories and unstructured comments [1]. Clinical relevance of PIs is evaluated using a multidimensional tool, named CLEOC (CLinical, Economic, and Organizational) [2]. The access of the relevance of the PIs is essential to demonstrate the added value of pharmacists. PIs are collected in a French national observatory Act-IPC. Nevertheless, only a third have their impact evaluated. In this work, we developed a clinical named entity recognition (NER) model to label medical expressions from unstructured comments recorded in PIs. Its performance was evaluated by comparing its output to a reference labelization. This is the first step to develop a predictive algorithm of clinical relevance of PIs.

${ }^{1}$ Corresponding Author, Justine CLARENNE, Pharmacy resident at the Pharmacy Department - Reims University Hospital, Rue du General Koenig, 51092 Reims, France; Email: clarenne.justine@gmail.com. 


\section{Methods}

A sample of 1087 PIs among 15233 PIs with clinical relevance was randomly selected from Act-IP ${ }^{\odot}$ (http://www.actip.sfpc.eu). Nine annotators - 8 expert pharmacists and a pharmacy resident - labeled medical expressions in the unstructured comments of the PIs. The quality of the annotations was evaluated for each medical label (inter annotator agreement - IAA - score). Theses labels were drug, dosage, treatment duration galenic form, route of administration, disease, germ, age, body surface area, kidney and kaliemia. The reference labelization referred to exact matches between two annotators (same label, start and end offset). The NER is rule-based using regular expressions. The regex were elaborated by using pre-elaborated lists of medical terms (ICD10) and without looking at the reference labelization. The output of the NER model was compared to the reference labelization. The performance of the NER model was evaluated with recall and precision.

\section{Results}

Four labels, drug, disease, dosage, and kidney were present in more than 500 PIs and were evaluated. IAA on the global labelization was superior to $70 \%$ for 7 seniors. For dosage and disease, IAA were respectively $60.8 \%$ and $54.5 \%$, which reveal an average agreement. The performance of the NER model showed a high precision for drug (77.5\%), dosage (78.4\%) and kidney (70.0\%). This also showed a high recall for drug (85.5\%), a mid-level recall for kidney (64.7\%) and a low recall for dosage (51.1\%). For disease, both precision (44.2\%) and recall (21.7\%) were considered low.

\section{Discussion and conclusions}

This study is the first to develop a NER to predict clinical relevance of PIs. It has been shown that clinical NER built on training data have better performances than those using rules [3]. The comparison between labelization proposed by the NER model and reference labelization allowed us to select three labels, drug, dosage, and kidney (high precision and correct recall) to mitigate any possible bias. The label disease has a low IAA score explained by an ambiguity among the pharmacists to define a disease. A larger dataset could achieve to select other labels and to enhance performance of the NER model [4]. The development of this clinical NER model will lead to create a predictive algorithm of clinical relevance of PIs.

\section{References}

[1] Allenet B, et al. Validation of an instrument for the documentation of clinical pharmacists' interventions. Pharm World Sci. 2006;28(4):181-8.

[2] Vo T-H. Evaluation of the potential impact of pharmacist interventions: development and validation of the CLEO multidimensional tool Available at: https://tel.archives-ouvertes.fr/tel-01315619, Accessed January 10, 2021.

[3] Lample G, et.al. Neural architectures for named entity recognition, arXiv [cs.CL] 2016.

[4] Lingren T, et.al. Evaluating the impact of pre-annotation on annotation speed and potential bias: natural language processing gold standard development for clinical named entity recognition in clinical trial announcements. J Am Med Inform Assoc. 2014 May-Jun;21(3):406-13. 